\title{
ON THE FAN AND LIDSKII MAJORISATIONS OF POSITIVE SEMIDEFINITE MATRICES
}

\author{
EUN-YOUNG LEE
}

Abstract. Let $A, B$ be two positive definite matrices. The Fan-Lidskii majorisations can be subsumed as the symmetric norm rearrangement inequalities

$$
\left\|A^{\downarrow}+B^{\uparrow}\right\| \leqslant\|A+B\| \leqslant\left\|A^{\downarrow}+B^{\downarrow}\right\|
$$

where the up/down arrows on $A, B$ mean the diagonal matrices with the same eigenvalues in decreasing/increasing order down to the diagonal. We refine these relations with sums of type $A+U B U^{*}$ and $A+V B V^{*}$ for two unitary matrices $U, V$ associated in a quite natural way to $A$ and $B$. Stronger results than majorisation are given by using simple averages in the unitary orbits of $A+B, A+U B U^{*}$ and $A+V B V^{*}$. Proofs rely on an orbital decomposition for positive block-matrices.

Mathematics subject classification (2010): 15A60, 47A30, 15A42.

Keywords and phrases: Matrix inequalities, positive definite matrices, majorisation, unitary orbit, trace class operators, matrix geometric mean.

\section{REFERENCES}

[1] T. Ando, Majorizations and inequalities in matrix theory, Linear Algebra Appl. 199 (1994), 17-67.

[2] R. Bhatia, Matrix Analysis (Springer, New-York, 1996).

[3] J.-C. Bourin AND F. HiaI, Norm and anti-norm inequalities for positive semi-definite matrices, Internat. J. Math. 22 (2011), 1121-1138.

[4] J.-C. BOURIN AND F. HIAI, Superadditive norm functionals on positive matrices and type $\mathrm{II}_{1}$ factors, preprint.

[5] J.-C. BOURIN AND E.-Y. LEE, Unitary orbits of Hermitian operators with convex and concave functions, Bull. London Math. Soc. 44 (2012), 1085-1102.

[6] J.-C. Bourin, E.-Y. LEE, M. Lin, Positive matrices partitioned into a small number of Hermitian blocks, Linear Algebra Appl. 438 (2013), 2591-2598.

[7] C.-K. LI, R. MATHIAS, The Lidskii-Mirsky-Wielandt theorem - additive and multiplicative versions, Numer. Math. 81 (1999), 377-413.

[8] B. Simon, Trace ideals and their applications, Second edition. Mathematical Surveys and Monographs 120. American Math. Soc., Providence, RI, 2005.

[9] X. ZHAN, The sharp Rado theorem for majorizations, Amer. Math. Monthly 110 (2003), 152-153. 